Finanzas y crecimiento en México: ¿Quién aporta más, la banca o la bolsa?

Lizethe Méndez-Heras, Francisco Venegas-Martínez y Ricardo Solis-Rosales 


\section{Finanzas y crecimiento en México: ¿Quién aporta más, la banca o la bolsa?}

Resumen: Este articulo estudia la relación entre la estructura financiera y el crecimiento económico de México durante el periodo 1980-2014. La literatura identifica dos tipos de estructura financiera: bank-basedy stock-market-based. En la primera, la banca comercial impacta positivamente la actividad económica y, en la segunda, el mercado bursátil influye en el desempeño de la economía. Una tercera visión considera que toda la actividad financiera (bancos, mercado bursátil y otras instituciones financieras) influye en el crecimiento. Estas hipótesis se evalúan mediante modelos de vectores de corrección de errores (VEC). Los hallazgos empiricos sugieren que, considerando la liquidez del sistema financiero, la actividad bursátil predominó durante todo el periodo de estudio; pero al considerar el tamaño del sistema financiero, prevaleció la actividad bancaria. También se muestra que el incremento de la liquidez. del sistema financiero elevó el crecimiento económico, pero el incremento del tamaño del sistema financiero disminuyó el PIB per cápita en el periodo 1980-2014. Y el análisis dinámico de corto plazo revela que si la estructura financiera se biciera más bursátil el efecto en el crecimiento económico sería positivo.

Palabras clave: estructura financiera, crecimiento económico, sector bancario, mercado bursátil.

Clasificación JEL: O16, G10, G20.

\section{Finance and Growth in Mexico: Who Contributes the Most: the Banks or the Stock Market?}

Abstract: This paper studies the relationship between the financial structure and the economic growth of Mexico during 1980-2014. The literature identifies two types of financial structure: bank-based and stock-market-based. In the first, commercial banking positively impacts economic activity, while in the second, the stock market influences the performance of the economy. A third view considers that all financial activity (banks, stock market and other financial institutions) influences growth. These bypotheses are assessed by using a VEC model. The empirical findings suggest that, considering the liquidity of the financial system, stock market activity predominates throughout the study period; but when we take the size of the financial system, banking activity prevails. We also show that increasing financial system liquidity had a positive effect on economic growth, although increasing the size of the financial system decreased the GDP per capita over the period 1980-2014. Moreover, the short-term dynamic analysis reveals that if the financial structure became more marketed-oriented, the effect on economic growth would be positive.

Keywords: financial structure, economic growth, banking sector, stock market.

https://doi.org/10.17533/udea.le.n96a344224

\section{(cC) BY-NC-SA}

Este artículo y sus anexos se distribuyen por la revista Lecturas de Economía bajo los términos de la Licencia Creative Commons Atribución-NoComercial-CompartirIgual 4.0. https://creativecommons.org/licenses/by-nc-sa/4.0/ 
Finance et croissance au Mexique: Qui contribue le plus, la banque ou la bourse? Résumé: Cet article étudie la relation entre la structure financière et la croissance économique du Mexique au cours de la période 1980-2014. La littérature identifie deux types de structure financière: bank-based et stock-market-based. Dans le premier cas, les services bancaires commerciaux ont un impact positif sur l'activité économique et, dans le second, le marché boursier influence la performance de l'économie. Un troisième point de vue considère que toutes les activités financières (banques, marchés boursiers et autres institutions financières) influencent la croissance. Ces hypothèses sont évaluées à l'aide de modèles vectoriels de correction d'erreur (VEC). Les résultats empiriques suggèrent que, compte tenu de la liquidité du système financier, l'activité boursière a prédominé tout an long de la période à l'étude; mais si l'on considère la taille du système financier, l'activité bancaire prévaut. Il montre également que l'augmentation de la liquidité du système financier a augmenté la croissance économique, mais l'augmentation de la taille du système financier a diminué le PIB par habitant au cours de la période 1980-2014. Et l'analyse dynamique à court terme révèle que si la structure financière devenait plus boursière, l'effet sur la croissance économique serait positif.

Mots clés: structure financière, croissance économique, secteur bancaire, marché boursier.

\section{Cómo citar / How to cite this item:}

Méndez-Heras, L. B., Venegas-Martínez, F., \& Solis-Rosales, R. (2022). Finanzas y crecimiento en México: ¿Quién aporta más, la banca o la bolsa?. Lecturas de Economía, 96, 235-278. https://doi.org/10.17533/udea.le.n96a344224 


\title{
Finanzas y crecimiento en México: ¿Quién aporta más, la banca o la bolsa?
}

\author{
Lizethe Méndez-Heras $\oplus^{a}$, Francisco Venegas-Martínez $\oplus^{\mathrm{b}}$ y Ricardo \\ Solís-Rosales $\oplus^{\mathrm{c}}$
}

-Introducción. -I. El nexo entre las finanzas y el crecimiento económico. -II. Desarrollo financiero en México. -III. Marco teórico y especificación de los modelos. -IV. Datos y su naturaleza -V. Análisis econométrico y especificación del modelo-Conclusiones. -Anexo.

-Referencias.

Primera versión recibida el 25 de octubre de 2020; versión final aceptada el 15 de julio de 2021

\section{Introducción}

La preocupación por la influencia que tiene el sistema financiero en el crecimiento se originó con el inicio del modo de producción capitalista. De acuerdo con Levine (1997) el pionero de este tipo de estudios fue Bagehot (1873), quién argumentó que el sistema financiero fue esencial para lograr la industrialización en Inglaterra durante el siglo XIX, al facilitar la movilización de capital —hipótesis que fue reforzada por Hicks (1969)—. Sin embargo, Schumpeter (1911) profundizó en el nexo de finanzas y el crecimiento y explicó que los servicios otorgados por los intermediarios financieros son esenciales para promover la inversión y el crecimiento económico porque permiten: 1) movilizar los ahorros; 2) invertir en proyectos; 3) administrar el riesgo, 4) monitorear la actividad empresarial; y 5) facilitar las transacciones.

a Lizethe Méndez-Heras: Docente Tiempo Completo, Universidad Ecotec, Facultad de Ciencias Económicas y Empresariales, Samborondón, Ecuador. Dirección electrónica: lmendez@ecotec.edu.ec https://orcid.org/0000-0002-3885-4584

b Francisco Venegas-Martínez: Profesor Investigador, Instituto Politécnico Nacional, Escuela Superior de Economía, Ciudad de México, México. Dirección electrónica: fvenegas1111@yahoo.com.mx https://orcid.org/0000-0003-1157-0298

c Ricardo Solis-Rosales: Profesor Investigador de la Universidad Autónoma MetropolitanaUnidad Iztapalapa, Facultad de Economía, Ciudad de México, México. Dirección electrónica: rsolis47@yahoo.com.mx https://orcid.org/0000-0002-5531-0570 
Méndez-Heras, Venegas-Martínez y Solis-Rosales: Finanzas y crecimiento en México...

Por su parte, Goldsmith (1969) fue pionero en medir la relación entre la actividad financiera y el crecimiento económico y creía que uno de los problemas más relevantes en el campo de las finanzas, es el efecto de la estructura financiera y del desarrollo financiero en el crecimiento económico. Al respecto, Demirgüç-Kunt y Levine (2001) señalan que Goldsmith tuvo tres resultados importantes: 1) fue exitoso en documentar la evolución del sistema financiero, en particular a los intermediarios financieros; 2) mostró que los bancos tienden a ser más grandes — con relación al Producto interno Bruto (PIB) — mientras más se desarrollan los países; 3) presentó evidencia de que los intermediarios financieros no bancarios y el mercado accionario crecen - en relación con los bancos- en tamaño e importancia. Asimismo, Goldsmith documentó una correlación positiva entre el desarrollo financiero y el nivel de actividad económica en 35 países utilizando datos de 1860 a 1963; sin embargo, se mostró poco dispuesto a proporcionar interpretaciones de causalidad. Por último, otra de las aportaciones de Goldsmith fue la distinción entre dos estructuras financieras: bancaria o bank-based y de mercado bursátil o stock-market-based.

La primera estructura, bank-based, sustenta que la actividad bancaria tiene un efecto positivo en el crecimiento económico porque los bancos: 1) optimizan la asignación del capital con base en la información sobre las empresas y los empresarios; 2) monitorean la gobernanza corporativa con mayor eficacia; 3) administran el riesgo de liquidez, cruzado e inter temporal, incrementando la eficiencia de las inversiones; 4) movilizan capital para desarrollar economías de escala; 5) forman relaciones de largo plazo con las empresas y no revelan información inmediatamente a los mercados, lo que permite indagar sobre las condiciones de mercado; y 6) monitorean a las empresas reduciendo el riesgo moral (Levine, 2002).

Levine (2005) explica que para varios autores ${ }^{1}$ los bancos más poderosos pueden ser más eficientes que un mercado de competencia, pues obligan a las empresas a pagar sus deudas — sobre todo en países con debilidad legal en el cumplimiento de los contratos-. Asimismo, Stiglitz (1985) enfatiza que el problema del free rider está implícito en los mercados

1 Como Gerschenkron (1962) y Rajan y Zingales (1998). 
atomizados y argumenta que "los mercados bursátiles más desarrollados revelan rápidamente información a los inversionistas y los disuade de destinar recursos a la investigación en las empresas. Por lo tanto, los mercados más desarrollados pueden obstruir los incentivos para identificar proyectos de innovación que fomenten el crecimiento económico" (Levine, 2002, p. 881).

El segundo enfoque, stock-market-based, señala que el mercado bursátil es mejor para el crecimiento económico que la actividad bancaria, porque es superior para financiar la creación de empresas y proyectos más riesgosos. De acuerdo con Levine (2002; 2005), esta visión es el contraataque al enfoque bank-based y se centra en los problemas generados por los bancos poderosos, pues asegura que el mercado es más efectivo para identificar, aislar y prevenir eficientemente la quiebra de las empresas, lo que evita daños en toda la economía.

Aunque existe un gran debate sobre qué estructura —-bancaria o bursátil— es la mejor para promover el crecimiento económico, Beck et al. (2001) aseguran que no es importante distinguir entre la estructura del sistema financiero y proponen otros dos enfoques alternativos para estudiar el efecto del sector financiero en el crecimiento económico: el de los servicios financieros, financial-services, y el legal, law and finance. Sobre el primero, señalan que ambos servicios financieros — los bancos y el mercado bursátil— son claves para la creación de empresas, la expansión industrial y el crecimiento económico. Mientras que el segundo, el legal, menciona que las leyes — sistema legal — son el principal determinante para que el sistema financiero facilite la innovación y el crecimiento económico.

En este artículo se realiza un esfuerzo para contribuir al debate sobre el efecto que tiene la estructura financiera en el crecimiento económico, particularmente en México. Para ello se plantean las siguientes preguntas de investigación: ¿Cuál fue la estructura financiera que predominó en México durante el periodo 1980-2014? ¿La estructura financiera mostró una relación significativa de largo plazo con el crecimiento económico durante el periodo de estudio? ¿Cuál fue el efecto del desarrollo financiero (bancos, mercado bursátil y otras instituciones financieras) en el crecimiento económico? 
Méndez-Heras, Venegas-Martínez y Solis-Rosales: Finanzas y crecimiento en México...

Las aportaciones de esta investigación con respecto de la literatura existente son: 1) para analizar el vínculo entre el desarrollo financiero y el crecimiento económico en México se estima la ecuación de Beck et al. (2001) mediante un modelo de vectores de corrección de errores (VEC); 2) se extiende el trabajo de Copelman (2000) al considerar la tasa de crecimiento del PIB, al incluir las variables de estructura y desarrollo financiero dentro del modelo, y al considerar un periodo de estudio más amplio; 3) los resultados empíricos obtenidos permiten proponer varias recomendaciones en materia de regulación sobre la actividad de los bancos comerciales y sus efectos adversos para el crecimiento económico —altos niveles de concentración de mercado y propensión a financiar empresas maduras-.

Otra contribución relevante de esta investigación consiste en construir los indicadores y tamaño de la estructura financiera en México, e incorporarlos como variables explicativas en un modelo VEC. Estos indicadores se han utilizado ampliamente para analizar la relación entre estructura financiera y crecimiento económico a nivel país, cross-country; sin embargo, existen pocos estudios para el caso mexicano (Copelman, 2000) y hasta donde se sabe, no hay algún trabajo sobre el tema que los incorpore.

Este artículo está organizado de la siguiente manera: en la sección I, se presenta una breve revisión de la literatura sobre el tema; a través de la sección II se plantea el marco teórico y la especificación del modelo econométrico; en el transcurso de la sección III se presentan los datos y se discute su naturaleza; en la sección IV se realizan diversas pruebas econométricas, se estima el modelo VEC y se realiza un análisis de funciones de impuso-respuesta y de descomposición de la varianza; por último, se proporcionan las conclusiones.

\section{El nexo entre las finanzas y el crecimiento económico}

A pesar de que la relación entre las finanzas y el crecimiento económico se ha estudiado desde hace muchos años, la evidencia empírica no ha sido concluyente y — por lo tanto- el tema aún sigue vigente. Además, los resultados no han podido corroborar que exista un efecto positivo de la estructura financiera —-bancaria o bursátil— en la actividad económica. El impacto positivo —o negativo— del sistema financiero en su conjunto, de la 
banca o del mercado de valores en el crecimiento depende en gran medida del periodo de estudio, de la unidad de análisis que se utilice para las estimaciones — varios países o un país_-, de las variables y de la metodología econométrica utilizada.

En la literatura empírica especializada sobre del tema destaca Beck et al. (2001), que utilizan tres metodologías para analizar la relación entre desarrollo financiero y crecimiento económico: 1) un modelo cross-country para 48 países de 1980 a 1995 que examina si los países crecen más en economías con estructura bancaria o bursátil; 2) un modelo cross-industry con datos panel para 34 países y 36 industrias de cada país de 1980 a 1989 que examina si las industrias que dependen fuertemente del financiamiento externo crecen más rápido que las que no dependen, y si influye en algo la estructura financiera en la creación de nuevas empresas; y, por último, 3) un análisis a nivel de empresa, cross-firm, usando datos sobre una selección de 33 países, de 1990 a 1995, para estudiar si las empresas tienen mayor probabilidad de crecer por arriba de su tasa estimada según la estructura financiera que poseen. Para los tres casos se estimaron modelos de mínimos cuadrados ordinarios (MCO) y variables instrumentales (VI). Sus principales conclusiones fueron: 1) los países no crecen más rápido en sistemas financieros con una estructura bancaria o con una estructura bursátil; 2) existe suficiente evidencia a favor de los enfoques de los servicios financieros o de la legalidad, pues se encontró que los países y las empresas crecen más rápido en economías con mayores niveles de desarrollo financiero en su conjunto — bancos y mercado accionario- y en países con sistemas legales más efectivos en proteger los derechos de los inversionistas.

En contraste con lo anterior, Tadesse (2002) realizó un estudio de 36 países, de 1980 a 1995, y encontró que los países dominados por pequeñas empresas crecen más rápido en los sistemas bank-based, mientras que los países con empresas grandes crecen más rápido en un sistema stock-market-based. Por su parte, Allen y Carletti (2008) se preguntaron si los sistemas de mercado $\mathrm{o}$ bancarios son mejores para proveer financiamiento a los proyectos y al desarrollo de nuevas tecnologías y sugieren que los sistemas financieros de mercado conducirán a mayor innovación que los sistemas bancarios. 
Méndez-Heras, Venegas-Martínez y Solis-Rosales: Finanzas y crecimiento en México...

Sobre el efecto que tiene el mercado bursátil en la actividad económica, Demirgüç-Kunt y Levine (1996) señalan que la rentabilidad de las acciones puede aumentar en el corto plazo, luego de la liberalización del control de capital, pero los países que están más abiertos a los flujos internacionales de capital tienden a tener mercados menos volátiles en el largo plazo que aquellos con controles de capital más estrictos. Por su parte, Arestis et al. (2001) estiman un modelo VAR para cinco países desarrollados y controlan los efectos de volatilidad en el sistema bancario y de mercado. Sus resultados muestran que, tanto el sistema bancario como el mercado de valores, tuvieron influencia en el crecimiento económico de Francia, Alemania y Japón; mientras que en Reino Unido y Estados Unidos la relación entre desarrollo financiero y crecimiento fue estadísticamente débil.

Entre los estudios de la última década, Beck et al. (2014) encuentran que existe una relación importante entre el tamaño del sistema financiero y el efecto de la actividad financiera en el crecimiento económico y señalan que "si el sector financiero es grande y no depende de la actividad 'tradicional' de intermediación (el negocio de la tasa de interés), podría generar mayor volatilidad en el crecimiento económico" (p. 14). Por su parte, Kim et al. (2016) estiman un modelo de sección cruzada para 41 países, con 1128 observaciones sobre el crecimiento del valor agregado de las industrias y encontraron que las industrias dominadas por pequeñas empresas crecen más rápido en un país con un sistema financiero bancario. Además, señalan que el efecto de la estructura financiera en el crecimiento de la industria se debe, principalmente, al crecimiento en el número de establecimientos, más que al crecimiento en el tamaño promedio de los establecimientos. Por su parte, Tongurai y Vithessonthi (2018) utilizaron una base de datos de panel con siete regiones ${ }^{2}$ durante el periodo $1960-2016$ y sus resultados muestran que la estructura bancaria tuvo un efecto negativo en la agroindustria en países con alto grado de desarrollo bancario. Méndez y Ongena (2019) estimaron mínimos cuadrados en dos etapas (MC2E) en 48 países y encontraron que la banca y el mercado de valores, en su conjunto, impactaron positivamente en el crecimiento económico de 1980 a 2014, incluso después de la crisis

2 Asia Oriental y Pacífico, Europa y Asia Central, Latinoamérica y el Caribe, Medio Oriente y Norte de África, Norte América, Asia del Sur y África Subsahariana. 
financiera internacional de 2008. Mientras que Demetriades y Rewilak (2020) analizaron 102 países de 1998 a 2017 y utilizaron datos panel para demostrar que el nexo entre las finanzas y el crecimiento puede recuperarse mediante el uso de medidas de desarrollo financiero ajustadas por calidad.

En un más reciente, Silva et al. (2021) utilizaron estimaciones con el método generalizado de momentos (GMM) con datos crediticios de Brasil y mostraron que el origen del crédito, la propiedad del banco, el tipo de crédito y el tipo de banco son importantes en el crecimiento económico. Además, encontraron que el crédito no destinado al sector empresarial se asocia con el crecimiento económico municipal y que, en general, el crédito proporcionado al sector empresarial por los bancos privados nacionales se correlaciona con tasas de crecimiento económico más altas.

También se ha encontrado evidencia empírica de que la relación entre la estructura financiera y el crecimiento económico es dinámica. Al respecto, Demir y Hall (2017) utilizaron un modelo no lineal autorregresivo de rezagos distribuidos para analizar la relación entre la estructura financiera y el desarrollo económico de Alemania, Estados Unidos, Francia y Turquía entre 1989 y 2012. Los autores encontraron que el desarrollo económico es relevante para determinar la estructura financiera, y que dicha relación no es estable, sino dinámica. Mientras que, Liu y Zhang (2020) estimaron un modelo de datos panel con 29 provincias de China de 1996 a 2013 y encontraron que la estructura financiera tiene efecto significativo en el crecimiento. Además, Chu (2020) empleó el método generalizado de momentos en 99 países de 1971 a 2015 y concluyó que, aunque prevalece la visión del mercado de valores, el efecto positivo se debilita significativamente si la estructura financiera está desequilibrada.

En cuanto a la evidencia para los países de América Latina, De Gregorio y Guidotti (1995) compararon los resultados de corte transversal de Barro (1991), quien utilizó una base de 100 países durante el periodo 1960-1985; con un panel de 12 países Latinoamericanos de 1960 a 1985. Sus resultados muestran que el crédito bancario al sector privado — como porcentaje del PIB - tuvo efecto negativo en el crecimiento económico de los países latinoamericanos. 
Cabe señalar que en este artículo nos centramos en la relación existente entre el sistema financiero y el crecimiento económico. Sin embargo, se ha encontrado evidencia sobre el efecto que tienen otras variables sobre la actividad económica de una nación, como la formación bruta de capital (Ortiz et al, 2019; Salazar-Nuñez \& Venegas-Martínez, 2018), el capital humano (Cuevas \& Calderón, 2020; Khan \& Chaudhry, 2019), la desigualdad de ingresos (Aiyar \& Ebeke, 2020; Sánchez, 2006), la pobreza (Acemoglu \& Robinson, 2013), por mencionar algunos. Y, aunque los determinantes del crecimiento económico de un país son diversos, este artículo representa un esfuerzo de ampliar la evidencia empírica sobre la relación entre la actividad financiera — bancaria y bursátil— en el crecimiento económico.

\section{Desarrollo financiero en México}

A continuación, se muestra la evolución del sistema financiero en México de 1980 a 2014. En los siguientes apartados, se observa que el sistema financiero de México se ha desarrollado a la par de la economía mexicana y se ha visto influenciado por sus auges y sus crisis.

\section{A. La década perdida}

De acuerdo con Colpeman (2001), la década de los años de 1980 ha sido conocida como "la década perdida" para muchos países de Latinoamérica y el caso de México no fue la excepción, con crisis del tipo de cambio, incumplimiento de la deuda externa y una severa desaceleración de la economía.

La crisis del tipo de cambio, acompañada de la caída de las reservas de divisas llevaron a México a incumplir con su deuda externa y a devaluar el peso en un $68 \%$ en 1982. Como resultado de la crisis, el PIB cayó 5,3\% y los salarios reales disminuyeron $32 \%$ entre 1982 y 1984" (p. 4). La autora comenta que a pesar de que se registró una leve recuperación entre 1984 y 1985, donde el PIB creció 3,7 \% y 2,8 \% respectivamente, una nueva la caída de los precios del petróleo envió a la economía de México a una crisis una vez más en 1986. 
Lo anterior motivó un proceso de ajuste con el objetivo de reducir la inflación, bajar el déficit presupuestario, liberalizar el comercio y reducir el papel del gobierno en la actividad económica a través de privatizaciones. Para ello se firmó el Pacto de Solidaridad Económica en 1987, con el objetivo de frenar el crecimiento de los salarios, llevar a cabo políticas monetarias y fiscales austeras, controlar ciertos precios clave de los servicios públicos y privados básicos y, lo que es más importante, fijar el tipo de cambio frente al dólar estadounidense y utilizarlo como ancla nominal de los precios. Adicionalmente, otras medidas se introdujeron al mismo tiempo, como liberalización comercial acelerada, la privatización de muchas empresas públicas y la liberalización financiera.

Para el caso del sector bancario, Clavellina (2013) señala que la creación de la banca múltiple fue un acontecimiento crucial en la década de los años de 1970, la cual comenzó operaciones en 1976. El objetivo era asegurar el financiamiento para la industria a mayores plazos, mejorar la asignación eficiente del crédito, generalizar el encaje legal, elevar la oferta de productos financieros y la vigilancia de las instituciones. Sin embargo, a finales de los años de 1970 la economía mexicana atravesó una serie de desequilibrios que causaron importantes afectaciones en las finanzas de los bancos comerciales, que culminaron en su estatización. Y fue a partir de 1988 que el país inició un proceso de reforma económico-financiera. Otro acontecimiento fue la renegociación de la deuda externa de 1988, que permitió recuperar la presencia de México en los mercados financieros internacionales. A partir de ese año se inició un nuevo periodo de expansión del crédito bancario al sector privado en México.

Por el lado del sector bursátil, Ejea et al. (1991) mencionan que al final del período 1978-1979, el mercado de valores presentaba cierto crecimiento respecto al nivel histórico, pero a la vez una tendencia al estancamiento relativo; un carácter sumamente concentrado y ligado a los bancos privados, la recomposición de su estructura, y un crecimiento de la operatividad con acciones.

Para el mercado de valores, la recuperación por el pago de la deuda pública se tradujo en una expansión durante esa década. El importe operado total entre 
Méndez-Heras, Venegas-Martínez y Solis-Rosales: Finanzas y crecimiento en México...

1978 y 1982 alcanzó el 18,1\% del PIB, mientras que entre 1982 y 1986 llegó a $70 \%$ del PIB. Ejea et al. (1991) explican que el crecimiento acelerado refleja la importancia del mercado de valores como espacio de financiamiento a los sectores institucionales de la economía, en detrimento del sistema bancario. Además, ese auge del mercado de valores permitió la obtención de utilidades extraordinarias por parte de los principales inversionistas y casas de bolsa.

\section{B. La euforia del crédito}

En la década de los años de 1990, destaca la crisis económica de 19941995, motivada por la debilidad del sistema bancario después de haber vivido su nacionalización en 1982, su reprivatización en 1991-1992 y la liberalización financiera de finales de los años de 1980. Cárdenas (2015) señala que, de diciembre de 1988 a noviembre de 1994, el crédito bancario aumentó a un ritmo promedio anual de casi $25 \%$ en términos reales, pues era la primera vez — en muchos años - que la población tenía acceso a crédito para adquirir todo tipo de bienes. Sin embargo, la gestión bancaria había perdido la capacidad de valuar riesgos y - aunado a la euforia general por las renegociaciones de la deuda y la firma del Tratado de Libre Comercio de América del Norte (TLCAN) - existía el deseo de colocar el mayor monto de crédito posible para recuperar la inversión realizada en la adquisición de los bancos, lo que motivó que la cartera vencida se cuadruplicara, al pasar de $14 \%$ a $53 \%$ entre 1991 y 1995. Aunado a lo anterior, los préstamos excedieron a los depósitos y el faltante fue cubierto por préstamos interbancarios, principalmente de bancos extranjeros y en moneda extranjera, lo que dejó muy expuesto al sistema bancario a cambios bruscos en el tipo de cambio y las tasas de interés (Cárdenas, 2015, p. 740).

Frente al aumento de la probabilidad de colapso del sistema financiero nacional, el gobierno implementó un conjunto de programas destinados al fortalecimiento del sector bancario en México. Estos programas se llevaron a cabo mediante el seguro de depósitos llamado FOBAPROA por 552300 millones de pesos en febrero de 1998, que se convertían en deuda pública. "Como consecuencia de esto, el rescate bancario fue cargado a cuenta de los contribuyentes” (Hernández \& Villagómez, 2013, p. 57). 
En suma, el periodo 1995-2000 fue de saneamiento de la banca y de modificaciones en las reglas de supervisión prudencial y en el fortalecimiento de la regulación. Después de la crisis financiera de 1994-1995, los bancos mexicanos contrajeron el crédito durante un periodo que concluyó hasta finales de esa década. Adicionalmente, se sumaron cambios institucionales y contables, por lo que "de 1995 al 2000 los bancos parecían más preocupados en alcanzar una situación financiera sana que en proporcionar crédito" (Negrín et al., 2010, p. 38).

Garrido y Martínez (2004) subrayan que, con la crisis de 1995 en México, el sistema financiero sufrió profundos cambios en dos dimensiones interrelacionadas. Por una parte, en la estructura institucional de propiedad de los principales agentes financieros y, por otra, en la configuración de los flujos y las condiciones de financiamiento. En el caso del mercado bursátil, la bolsa mexicana de valores modernizó sus operaciones, con un sistema electrónico y un listado de instrumentos internacionales; sin embargo, Ortiz et al. (2007) detallan que el mercado accionario mexicano era aún muy pequeño; la oferta y la demanda de títulos accionarios era muy limitada. Además, no se había constituido en una fuente importante de financiamiento corporativo. El grueso de la negociación de acciones en el mercado bursátil mexicano se concentra en pocas empresas y predomina excesivamente el mercado secundario, por lo que los recursos que allí se movilizan no constituyen financiamiento fresco para las empresas, sino que obedecen a decisiones de portafolio de los inversionistas. Además, los autores señalan que, en 1990, los flujos de capital externo que ingresaron al mercado accionario representaron el 75,74\% de los flujos de inversión extranjera directa, y en 1991, 1992 y 1993, respectivamente, el 132,98\%, el 108,89\% y el 244,18\%. Además, la inversión extranjera en términos de valor de mercado se incrementó de alrededor del 13\% en 1989 a cerca del $80 \%$ en 1993, generando mayor volatilidad y fragilidad de la bolsa y la economía en general.

En resumen, la desregulación y liberalización de los mercados financieros de México han motivado el ingreso de cuantiosos flujos de capital externo. Sin embargo, la incertidumbre de estos, su falta de arraigo y limitado financiamiento a las inversiones reales son factores determinantes para la presencia de una marcada fragilidad financiera, que transforma —en 
particular - al mercado accionario en posible motor y transmisor de severas crisis, "que a su vez, aunadas a las crisis cambiarias y bancaria, pueden someter al país a la gestación de nuevas crisis, tal como ocurrió en 1994-1995" (Ortiz et al., 2007, p. 134).

\section{El siglo $X X I$}

En la primera década de este, el sistema financiero nacional e internacional se vio afectado por la crisis de los créditos hipotecarios en Estados Unidos que estalló en 2007 y que, por diversas razones, se convirtió en crisis financiera internacional. Entre los efectos de la crisis en la economía real se registraron "caídas en el producto económico mundial, en las bolsas mundiales y en el comercio internacional, que fueron agudos desde octubre de 2008 y que tocaron piso hacia agosto de 2009" (Marichal, 2010, p. 316). En México, la caída más pronunciada se registró en el segundo trimestre de 2019 que, según datos del del Instituto Nacional de Estadística y Geografía (INEGI), fue de $-7,7 \%$ respecto al mismo trimestre del año anterior.

Ambos sectores —el bancario y el bursátil— se vieron afectados no solamente en México, sino en todo el mundo. En la Figura 1 se puede observar la caída en el crédito nacional privado que ofrecen los bancos y en las acciones negociadas en la bolsa de valores, ambos como porcentaje del PIB. Y, de la mano con la recuperación de la economía, el sistema financiero en su conjunto logró una recuperación para el año 2014.

A través de los eventos más relevantes durante el periodo de estudio, se ha podido describir que la actividad económica tiene influencia en el sistema financiero, pero que las instituciones que conforman el sistema financiero también influyen en el crecimiento económico, en algunos casos de forma positiva y en otros de manera negativa. Por esa razón, es relevante cuantificar el efecto de largo plazo que tienen la actividad bancaria y bursátil en la economía mexicana. 
Figura 1. Desarrollo del Sistema Financiero Mexicano 1980-2014 (valores como \%PIB)

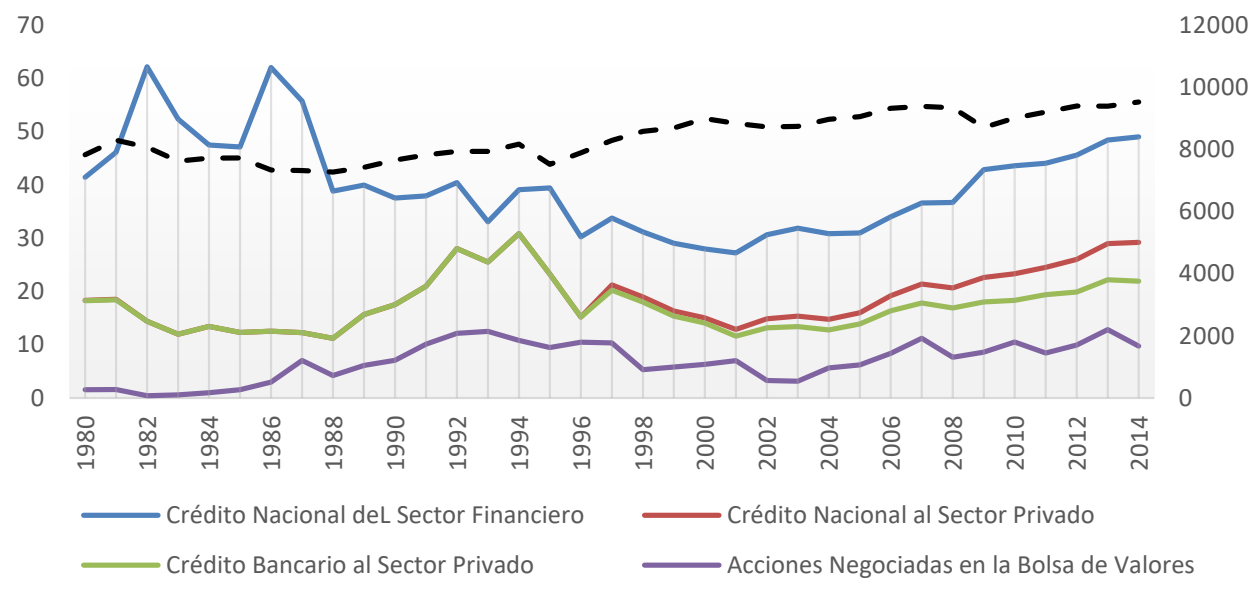

- - PIB Per Cápita

Fuente: elaboración propia con datos de Banco Mundial (2016) y GFDD Banco Mundial (2017).

\section{Marco teórico y especificación de los modelos}

Para justificar la relación entre la estructura financiera y el crecimiento económico se plantea, a continuación, el modelo neoclásico estándar de Solow (1956) donde la producción agregada está en términos de la tasa de crecimiento de la productividad total de los factores, PTF, la cual es explicada por factores diferentes al trabajo y al capital. Bajo el supuesto de una función de producción del tipo Cobb-Douglas con rendimientos constantes a escala se puede escribir:

$$
Y_{t}=A_{t} K_{t}^{\alpha} L_{t}^{1-\alpha}
$$

donde $Y_{t}$ es la producción, $K_{t}$ es el capital ${ }^{3}, L_{t}$ es el trabajo y $A_{t}$ es el coeficiente tecnológico. Mientras que $\alpha$ y $1-\alpha$ son las participaciones del capital y del trabajo en el producto, respectivamente. Ahora es posible explicar

3 En este artículo, la formación bruta de capital es considerada como proxy del capital. 
Méndez-Heras, Venegas-Martínez y Solis-Rosales: Finanzas y crecimiento en México...

la PTF, como la tasa residual entre la tasa autónoma y las tasas de crecimiento del capital y del trabajo mediante la relación:

$$
P T F=x_{A}-\alpha x_{K}-(1-\alpha) x_{L},
$$

donde $x_{A}$ es la tasa autónoma de crecimiento del producto, $x_{K}$ es tasa de crecimiento del capital, y $x_{L}$ es la tasa de crecimiento del trabajo. Así, entre estos factores que explican la PTF se encuentran la eficiencia de la estructura financiera, el avance tecnológico, la inversión en investigación y desarrollo, las patentes, las exportaciones de productos de alto contenido tecnológico, etcétera. Por lo tanto, bajo los supuestos anteriores, se espera que en el largo plazo la estructura financiera explique la tasa de crecimiento del PIB.

Una vez establecido el marco teórico de la PTF se plantea la especificación de modelo VEC para la presente investigación, la cual se basa en el marco teórico anterior y en la metodología propuesta por Beck et al. (2001), quienes estiman una ecuación de crecimiento de la siguiente forma:

$$
\text { Growt }_{t}=\alpha^{\prime} X_{t}+\beta F D_{t}+\gamma F S_{t}+\varepsilon_{t},
$$

donde Growth $_{t}$ es la tasa promedio anual de crecimiento del PIB real, $X_{t}$ es un conjunto de potenciales determinantes de crecimiento, $F D_{t}$ es un conjunto de indicadores de desarrollo financiero, $F S$ es un conjunto de indicadores de estructura financiera y $\varepsilon_{t}$ es un término de error. El modelo de VEC tiene la forma siguiente:

$$
\begin{gathered}
\Delta Y_{t}=B_{0}+\Pi Y_{t-i}+\Gamma_{1} \Delta Y_{t-1}+\Gamma_{2} \Delta Y_{t-2}+ \\
\cdots+\Gamma_{(p-1)} \Delta Y_{t-(p-2)}+w_{t},
\end{gathered}
$$

donde $Y_{t}$ es un vector de $n \times 1$ variables en el tiempo $t, B_{0}$ representa un vector de términos de intercepción de dimensión $n \times 1,\left\{B_{i}, i=1,2, \ldots, p\right\}$ y $\left\{B_{j}, j=2,3, \ldots, p+1\right\}$ representan matrices de coeficientes de $n \times n$, $p$ es el máximo de rezagos incluidos en el modelo, $w_{t}$ es un vector de $n \times 1$ innovaciones que se comportan como ruido blanco (con media cero y varianza constante), $n$ el número de variables en el modelo, $\Pi=\sum_{i=1}^{p} B_{i}-I$ y $\Gamma_{i}=\sum_{j=i+1}^{p} B_{j}$. De acuerdo con la cuarta implicación del teorema de 
la representación de Granger, si un vector $k$-dimensional de variables $I(1)$ involucra a una o más relaciones de cointegración, entonces existe un modelo VEC que se puede representar satisfactoriamente por la ecuación 4 (Cuevas, 2010). Con el objetivo de medir la relación de largo plazo entre la estructura financiera y el crecimiento económico se estiman las relaciones:

$$
\begin{gathered}
Y_{t}=\left[P I B p c_{t}, F S_{t}, X_{t}\right] \\
Y_{t}=\left[P I B p c_{t}, F D_{t}, X_{t}\right],
\end{gathered}
$$

donde $P I B p c_{t}$ es el PIB per cápita real en el tiempo $t, F S_{t}$ es un conjunto de indicadores de estructura financiera, $F D_{t}$ es un conjunto de indicadores de desarrollo financiero y $X_{t}$ es un conjunto de potenciales determinantes de crecimiento.

\section{Datos y su naturaleza}

En primer lugar, se hace énfasis en los indicadores de desarrollo financiero y de estructura financiera para México de 1980 a 2014. Los indicadores se elaboraron con la metodología propuesta por Beck et al. (2001) con datos anuales del Banco Mundial (2016). Estos indicadores servirán para mostrar cuál fue la estructura financiera que predominó en México durante el periodo de estudio. Primero, se elaboraron dos variables de estructura financiera (FS, financial structure):

a) Estructura financiera ( $S A$, structure-activity), que compara el valor de las acciones negociadas en el mercado bursátil con respecto a los créditos otorgados por los bancos comerciales (Figura 2, panel a)

b) Tamaño de la estructura financiera ( $S S$, structure-size), compara el tamaño del mercado bursátil (capitalización) con respecto del crédito otorgado por los bancos comerciales (Figura 2, panel b)

A su vez, se obtuvieron dos variables de desarrollo financiero $(F D$, financial development): 
Méndez-Heras, Venegas-Martínez y Solis-Rosales: Finanzas y crecimiento en México...

c) Actividad financiera ( $F A$, financial-activity), que representa la actividad financiera en su conjunto: bancos, mercado bursátil y otras instituciones financieras (Figura 2, panel c)

d) Tamaño de las instituciones financieras (FS, financial-size), que considera el tamaño de todo del mercado bursátil, los créditos otorgados por los bancos y los créditos otorgados por otras instituciones financieras (Figura 2, panel d).

Figura 2. Indicadores de estructura y desarrollo financiero en México

a. Estructura financiera (SA)

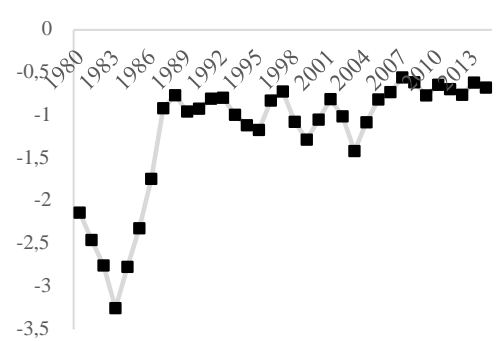

c. Actividad financiera (FA)

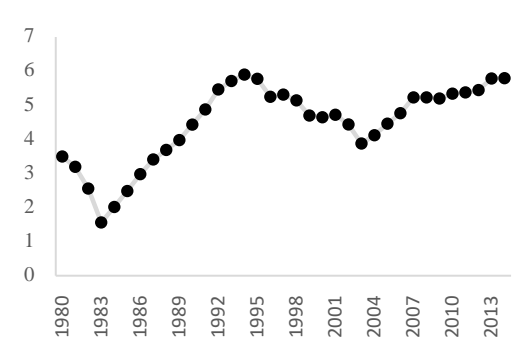

b. Tamaño de la estructura financiera (SS)

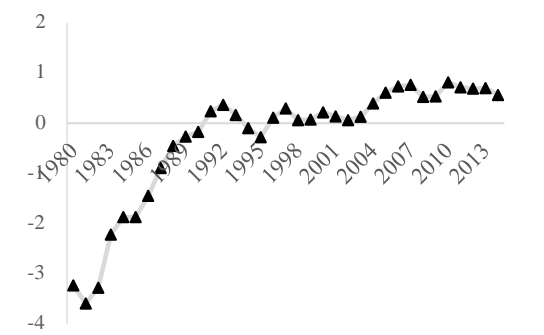

d. Tamaño de las instituciones financieras

(FS)

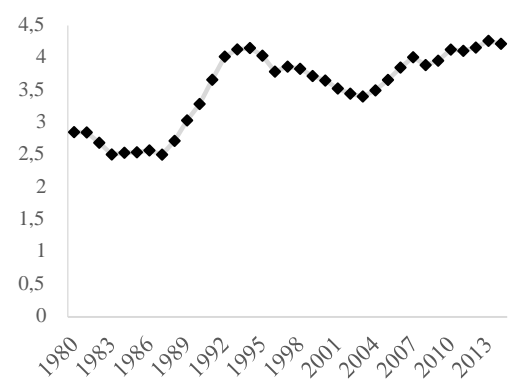

Fuente: elaboración propia con datos de Banco Mundial (2016) y GFDD Banco Mundial (2017).

Como se puede observar en el panel a de la Figura 2, al considerar la liquidez del mercado accionario, la actividad bancaria fue mayor que la actividad bursátil, pues el indicador tiene signo negativo. Mientras que al considerar el tamaño del mercado accionario —el valor de capitalización de 
las empresas que cotizan en bolsa-, se puede ver que en la década de los años de 1990 empieza a predominar la actividad de mercado bursátil ya que el signo comienza a ser positivo. Con respecto a los paneles b y d, al considerar el tamaño de las instituciones financieras y el tamaño de la estructura financiera, se observa que la actividad bancaria predominó hasta inicios de los años de 1990, y luego comenzó a destacar la actividad financiera — bursátil— hasta la fecha, de acuerdo con el panel c.

Beck et al. (2009) explican que, mientras más altos sean los valores de estructura financiera $(S A)$ y tamaño de la estructura financiera $(S S)$, predominará la estructura financiera bursátil. La diferencia radica en que el primero se enfoca en el total de acciones en circulación en la bolsa, mientras que el segundo se enfoca en la liquidez de la bolsa de valores (Beck et al., 2009). Los autores obtuvieron los valores promedios de ambos indicadores para cinco años entre 1983 y 2007, en 79 países clasificados en grupos por nivel de ingresos; y encontraron que la relación entre el mercado de valores y la actividad bancaria se incrementó durante los 25 años de estudio, para todos los grupos de ingreso. También observaron que, mientras el indicador de $S S$ no varió mucho entre diferentes grupos de ingreso, el indicador $S A$ fue mayor en los países con alto nivel de ingresos. En dicho estudio, los autores encontraron que el indicador $S A$ registró un incremento en los países de América Latina y el Caribe de 1988 a 1993, pero disminuyó de 1993 al 2007. Sin embargo, para el caso de México el indicador $S A$ fue negativo en todo el periodo, lo que implica que predominó la actividad bancaria (Figura 2, panel a). Lo anterior coincide con que países de bajos ingresos suelen tener una estructura financiera $(S A)$ bancarizada.

En la Tabla A1 del anexo se proporciona la descripción completa de todas las variables que intervienen en esta investigación como el PIB per cápita real (PIBpc) y otros determinantes del crecimiento económico: inflación (Inf), gasto de gobierno (Gov) y comercio (Com). Todas las series se obtuvieron del Banco Mundial (2016). ${ }^{4}$ La Tabla 1 muestra la estadística descriptiva de las variables analizadas. Dentro del periodo de estudio, la media del crecimiento económico del PIB per cápita fue de $0,90 \%$, la inflación de $0,28 \%$, el

4 Adicionalmente, Beck et al. (2001) consideran el mercado negro o black market premium, que en esta investigación no se considera por falta de datos para todo el periodo de estudio. 
Méndez-Heras, Venegas-Martínez y Solis-Rosales: Finanzas y crecimiento en México...

gasto de gobierno de 10,77\% y las exportaciones netas de 44,77\%. Por su parte, los valores promedio de la estructura financiera y el tamaño de la estructura financiera fueron de $-1,20$ y $-0,31$, respectivamente. Las medias de la actividad financiera y el tamaño de la actividad financiera fueron de 4,47 y 3,52, respectivamente. En el año de 1980 se registró el nivel máximo de crecimiento del 6,65\%; mientras que el nivel mínimo fue de -7,52\% en 1995. Los valores mínimo y máximo de la estructura financiera fueron de $-3,25$ en 1983 y de $-0,55$ en 2007; mientras que el tamaño de la estructura financiera alcanzó los valores mínimo y máximo de -3,56 en 1982, y de 0,81 en 2010. Por su parte, la actividad financiera obtuvo el mínimo de 1,56 en 1983 y el máximo de 5,90 en 1994; mientras que el tamaño de la actividad financiera alcanzó el mínimo de 2,51 en 1987 y el máximo de 4,26 en 2013. En cuanto a las variables potenciales de crecimiento económico, la inflación mínima fue en $2013(0,02 \%)$ y la máxima en 1987 (1.40\%). El gasto de gobierno más bajo fue en 1989 (8,27\%) y el máximo en 1995 (13,17\%). Por último, las exportaciones netas alcanzaron un mínimo en 1981 del 23,34\% y un máximo en 2013 del 66,41\%.

Tabla 1. Estadística Descriptiva de las variables bajo estudio

\begin{tabular}{lccccc}
\hline & PIBpc & $F A$ & $F S$ & $S A$ & $S S$ \\
\hline Media & 0,90 & 4,47 & 3,52 & $-1,20$ & $-0,31$ \\
Mediana & 1,60 & 4,72 & 3,66 & $-0,92$ & 0,13 \\
Max. & 6,65 & 5,90 & 4,26 & $-0,55$ & 0,81 \\
Min. & $-7,52$ & 1,56 & 2,51 & $-3,25$ & $-3,59$ \\
Desv. Est. & 3,37 & 1,16 & 0,59 & 0,71 & 1,23 \\
Asimetría & $-0,90$ & $-0,86$ & $-0,57$ & $-1,54$ & $-1,49$ \\
Curtosis & 3,50 & 2,83 & 1,87 & 4,19 & 4,09 \\
Obs. & 35 & 35 & 35 & 35 & 35 \\
\hline
\end{tabular}

Fuente: elaboración propia con datos de Banco Mundial (2016) y GFDD Banco Mundial (2017). 


\section{Análisis econométrico y especificación del modelo}

A continuación, se presentan varias pruebas sobre la estacionariedad, cointegración y causalidad en el sentido de Granger. Posteriormente, se estima el modelo VEC y se realiza un análisis de funciones de impuso-respuesta y de descomposición de la varianza.

\section{A. Pruebas de raíces unitarias y cointegración}

Para corroborar que las series de tiempo son integradas de orden uno, $I(1)$, se realizaron tres pruebas a las variables en niveles y en primeras diferencias: Dickey-Fuller Aumentada, Phillip-Perron y la prueba de KiwiatkowskiPhillips-Schmidt-Shin (KPSS). Asimismo, se utilizó el método de Hamilton (1994) para determinar si la serie presenta una constante y una tendencia lineal (I-TD) en la ecuación de prueba, o solo una constante (I), esto permite elegir la especificación más adecuada de los datos ${ }^{5}$. Las pruebas se realizaron para los valores en niveles y en primeras diferencias (Tabla 2) y las pruebas de raíces unitarias conjuntas Levin-Lin-Chu se presenta en las tablas A2 y A3 del anexo.

Las tablas 3 y 4 presentan las pruebas de cointegración de Johansen (1995). Como se puede observar, en el modelo 1 (M1) existen tres ecuaciones de cointegración a un nivel de significancia de $5 \%$ y para el modelo 2 (M2) también existen tres ecuaciones de cointegración al $5 \%$ de significancia.

\section{B. Pruebas de causalidad de Granger}

La causalidad de Granger mide la correlación de los valores pasados de una variable con los valores presentes de otras variables en el modelo. En el Tabla 5 se observa que los valores pasados de la tasa de crecimiento económico explican los valores presentes de la actividad de la estructura financiera $(S A)$, del tamaño de la estructura financiera $(S S)$ y de la actividad financiera $(F A)$.

5 Véase, al respecto, Cuevas (2010). 
Méndez-Heras, Venegas-Martínez y Solis-Rosales: Finanzas y crecimiento en México...

Tabla 2. Pruebas de raiz unitaria y estacionariedad (1980-2014)

\begin{tabular}{|c|c|c|c|c|c|}
\hline Variable & $\begin{array}{c}\text { Especificación } \\
\text { de la ecuación } \\
\text { de prueba }\end{array}$ & $\begin{array}{c}\text { Estadístico de } \\
\text { la prueba ADF } \\
\text { ( } H_{0}: \text { Raíz } \\
\text { unitaria })\end{array}$ & $\begin{array}{c}\text { Estadístico de } \\
\text { la prueba PP } \\
\left(H_{0}: \text { Raíz }\right. \\
\text { unitaria })\end{array}$ & $\begin{array}{c}\text { Estadístico de la } \\
\text { prueba KPSS }\left(H_{0} \text { : }\right. \\
\text { Estacionariedad })\end{array}$ & $\begin{array}{l}\text { Orden de } \\
\text { integración }\end{array}$ \\
\hline$P I B p c$ & I-TD & $-2,522001$ & $-2,203945$ & 0,147793 & $I(0)$ \\
\hline$\triangle P I B p c$ & I & $-5,862496^{* * *}$ & $-5,864088^{* * *}$ & $0,183692^{*}$ & $I(1)$ \\
\hline$S A$ & I & $-2,525918^{* * *}$ & $-1,600628$ & 0,502616 & $I(0)$ \\
\hline$\Delta S A$ & I & $-4,356963^{* * *}$ & $-3,675598^{* * *}$ & $0,072895^{*}$ & $I(1)$ \\
\hline SS & I-TD & $-3,492567^{* * *}$ & $-1,525389$ & 0,173176 & $I(0)$ \\
\hline$\Delta S S$ & I & $-4,639012^{* * *}$ & $-4,111910^{* * *}$ & 0,439189 & $I(1)$ \\
\hline$F A$ & I & $-1,776664$ & $-1,123430$ & 0,462042 & $I(0)$ \\
\hline$\Delta F A$ & I & $-3,349308^{* *}$ & $-3,356454^{* *}$ & $0,086635^{*}$ & $I(1)$ \\
\hline FS & I-TD & $-3,373953^{*}$ & $-1,930678$ & $0,100072^{*}$ & $I(0)$ \\
\hline$\Delta F S$ & I & $-2,807688^{*}$ & $-2,859517^{*}$ & $0,073262^{*}$ & $I(1)$ \\
\hline $\operatorname{Inf}$ & I-TD & $-1,967271$ & $-3,126471$ & $0,101952^{*}$ & $I(0)$ \\
\hline$\Delta \operatorname{Inf}$ & I & $-5,533136^{* * *}$ & $-8,866625^{* * *}$ & 0,5 & $I(1)$ \\
\hline Gov & I & $-1,405016$ & $-1,516527$ & 0,383203 & $I(0)$ \\
\hline$\Delta G o v$ & I & $-5,186046^{* * *}$ & $-5,186046^{* * *}$ & $0,080570^{*}$ & $I(1)$ \\
\hline Com & I-TD & $-4,141150^{*}$ & $-2,425912$ & $0,040390^{*}$ & $I(0)$ \\
\hline$\Delta \mathrm{Com}$ & I & $-4,985548^{* * *}$ & $-8,306887^{* * *}$ & 0,241802 & $I(1)$ \\
\hline
\end{tabular}

Nota: $\mathrm{I}=$ Intercepto, I-TD = intercepto y tendencia. Pruebas: ADF = Dickey-Fuller Aumentada; PP = Phillips Perron; KPSS = Kwiatokowski, Phillips, Schmidt y Shin.

Rechazo de la hipótesis nula a los niveles: ${ }^{*} 10 \%$ de significancia estadística, ${ }^{* *} 5 \%$ de significancia estadística, *** $1 \%$ de significancia estadística.

Fuente: elaboración propia con datos de Banco Mundial (2016) y GFDD Banco Mundial (2017). 
Tabla 3. Pruebas de cointegración de Johansen sobre la base de la traza (M1)

\begin{tabular}{lccc}
\hline Hipótesis nula & Hipótesis alternativa & Estadístico de la traza & Valor crítico 5\% \\
\hline$r=0$ & $r \geq 1$ & 77,80220 & $29,79707^{*}$ \\
$r \leq 1$ & $r \geq 2$ & 24,87533 & $15,49471^{*}$ \\
$r \leq 2$ & $r \geq 3$ & 4,803361 & $3,841466^{*}$ \\
\hline
\end{tabular}

Nota: $r$ denota el número de ecuaciones de cointegración. Un asterisco * indica rechazo de la hipótesis nula al nivel de significación del $5 \%$, dados los valores críticos elaborados por MacKinnon, Haug y Michelis (1999). Pruebas de la traza indican la existencia de 3 ecuaciones de cointegración al nivel de significación de $5 \%$.

Fuente: elaboración propia con datos de Banco Mundial (2016) y GFDD Banco Mundial (2017).

Tabla 4. Pruebas de cointegración de Johansen sobre la base de la traza (M2)

\begin{tabular}{lccc}
\hline Hipótesis nula & Hipótesis alternativa & Estadístico de la traza & Valor crítico 5\% \\
\hline$r=0$ & $r \geq 1$ & 65,13493 & $29,79707^{*}$ \\
$r \leq 1$ & $r \geq 2$ & 20,49661 & $15,49471^{*}$ \\
$r \leq 2$ & $r \geq 3$ & 5,584821 & $3,841466^{*}$ \\
\hline
\end{tabular}

Nota: $r$ denota el número de ecuaciones de cointegración. Un asterisco * indica rechazo de la hipótesis nula al nivel de significación del $5 \%$, dados los valores críticos elaborados por MacKinnon, Haug y Michelis (1999). Pruebas de la traza indican la existencia de 3 ecuaciones de cointegración al nivel de significación del $5 \%$.

Fuente: estimaciones de los autores a partir de los datos de Banco Mundial (2016).

Las siguientes pruebas corroboran que los resultados son confiables y tienen el objetivo de evaluar que los errores se distribuyen de manera normal, por lo que son homoscedásticos y no presentan autocorrelación ni correlación serial, y además son estables para ambos modelos. La Tabla 6 resume los resultados, y en las tablas del anexo - A4 hasta A8 - se presentan en detalle todas las pruebas realizadas; con lo cual se puede concluir que los errores en ambos modelos se comportan como ruido blanco.

Por último, en la sección de pruebas de diagnóstico, en la Tabla A10 del anexo, se presenta la prueba relativa a la condición de estabilidad del modelo de 1980 a 2014, así como las raíces inversas del polinomio autorregresivo característico de ambos modelos. 
Méndez-Heras, Venegas-Martínez y Solis-Rosales: Finanzas y crecimiento en México...

Tabla 5. Pruebas de causalidad en el sentido de Granger

\begin{tabular}{lccc}
\hline Muestra: $1980-2014$ & Obs. & Estadístico $F$ & Prob. \\
\hline Hipótesis nula: & 32 & Rezagos: 2 & \\
SA no causa a la Granger PIBpc & 32 & 0,5299 & 0,5947 \\
PIBpc no causa a la Granger $S A$ & 32 & 3,47637 & 0,0454 \\
SS no causa a la Granger PIBpc & 32 & 0,17616 & 0,8394 \\
PIBpc no causa a la Granger $S S$ & 32 & 3,48452 & 0,0451 \\
FA no causa a la Granger PIBpc & 32 & 0,06322 & 0,9389 \\
PIBpc no causa a la Granger FA & 32 & 2,95226 & 0,0693 \\
FS no causa a la Granger PIBpc & 32 & 1,67072 & 0,207 \\
PIBPc no causa a la Granger FS & 32 & 2,32312 & 0,1172 \\
\hline
\end{tabular}

Fuente: elaboración propia con datos de Banco Mundial (2016) y GFDD Banco Mundial (2017).

Tabla 6. Pruebas de diagnóstico de los residuos de la regresión, 1980-2014

\begin{tabular}{lll}
\hline & \multicolumn{2}{c}{ Modelo 1 (M1) } \\
\hline Tipo de prueba & Hipótesis nula & Valor de probabilidad \\
Correlación serial (a) & No hay correlación serial* & 0,7556 \\
Heteroscedasticidad (b) & Homoscedasticidad & 0,7089 \\
Normalidad (c) & Normalidad & 0,9876 \\
\hline Tipo de prueba & Modelo 2 (M2) & \\
Correlación serial (a) & Hipótesis nula & Valor de probabilidad \\
Heteroscedasticidad (b) & Homoscedasticidad & 0,4442 \\
Normalidad (c) & Normalidad & 0,0904 \\
\hline
\end{tabular}

Nota: Pruebas: a) Breusch-Godfrey hasta el orden de rezago 4, b) White con dos rezagos, c) Jarque-Bera. ${ }^{*}$ Hasta el tercer rezago.

Fuente: estimaciones de los autores a partir de los datos de Banco Mundial (2016) y GFDD Banco Mundial (2017). 


\section{Resultados empiricos: el modelo VEC}

La Tabla 7 presenta las ecuaciones de cointegración de los dos modelos estimados, que son significativas al 5\%. En el primer modelo (M1), los coeficientes de la estructura financiera $(S A)$ y tamaño de la estructura financiera $(S S)$ son estadísticamente significativos ya que el estadístico $t$ es mayor, a \pm 2 . Por lo tanto, se puede decir que existe una relación de largo plazo entre la estructura financiera y el crecimiento económico de México. Mientras que en el segundo modelo (M2), los coeficientes de la actividad financiera (FA) y del tamaño de la actividad financiera (FS) también son estadísticamente significativos y, por lo tanto, existe una relación de equilibrio de largo plazo entre la actividad financiera en su conjunto y el PIB per cápita de México. En ambos modelos, las variables potenciales de crecimiento económico se definen como variables exógenas que captan efectos macroeconómicos de la inflación (Inf), el gasto de gobierno (Gov) y las exportaciones netas (Com).

En el modelo 1, el signo positivo del coeficiente $S A$ implica que al evaluar la liquidez de la estructura financiera predominó la actividad bursátil. Y, el signo negativo del coeficiente $S S$, muestra que considerando el tamaño de la estructura financiera dominó la actividad bancaria.

Los resultados del modelo 2, confirman que el incremento en la liquidez del sistema financiero $(F A)$, que representa la actividad financiera de bancos, mercado y otras instituciones financieras; eleva el crecimiento económico. Ese aumento puede responder a incrementos de los créditos bancarios, de las acciones negociadas en el mercado de valores, de los créditos de otras instituciones financieras no bancarias.

Finalmente, el incremento en el tamaño de las instituciones financieras $(F S)$, que representa el tamaño de las instituciones financieras; reduce el crecimiento económico. Ese incremento puede ser resultado del aumento en la capitalización del mercado financiero o el aumento de los créditos bancario y no bancario. Este último resultado coincide con lo planteado por Beck et al. (2014), quienes advierten sobre la relación entre un sector financiero grande, que no depende de la actividad tradicional de intermediación y asociado a mayores niveles de volatilidad en el crecimiento económico. 
Méndez-Heras, Venegas-Martínez y Solis-Rosales: Finanzas y crecimiento en México...

Tabla 7. Coeficientes de los modelos VEC

Vector de Corrección de Errores

Muestra: 1983-2014. Observaciones incluidas: 32 después de ajustes

\begin{tabular}{|c|c|c|c|c|}
\hline & \multicolumn{2}{|c|}{ Modelo 1} & \multicolumn{2}{|c|}{ Modelo 2} \\
\hline & $\begin{array}{l}\text { Ecuación de } \\
\text { Cointegración }\end{array}$ & EE y T & $\begin{array}{l}\text { Ecuación de } \\
\text { Cointegración }\end{array}$ & EE y T \\
\hline$\triangle P I B p c(-1)$ & 1 & & 1 & \\
\hline \multirow[t]{2}{*}{$\Delta S A(-1)$} & 0,439613 & $(0,05027)$ & - & - \\
\hline & & {$[8,74461]$} & & - \\
\hline \multirow[t]{2}{*}{$\Delta S S(-1)$} & $-0,369819$ & $(0,05746)$ & - & - \\
\hline & & {$[-6,43630]$} & & - \\
\hline \multirow[t]{2}{*}{$\Delta F A(-1)$} & - & - & 0,096914 & $(0,01340)$ \\
\hline & & - & & {$[7,23391]$} \\
\hline \multirow[t]{2}{*}{$\Delta F S(-1)$} & - & - & $-0,236256$ & $(0,03496)$ \\
\hline & & - & & {$[-6,75807]$} \\
\hline$C$ & 0,01076 & & $-0,004537$ & \\
\hline $\begin{array}{l}\text { Determinante de Cova- } \\
\text { rianza Residual }\end{array}$ & $1,77 \mathrm{E}-07$ & & $1,22 \mathrm{E}-07$ & \\
\hline Log de Verosimilitud & 109,0410 & & 114,8440 & \\
\hline $\begin{array}{l}\text { Criterio de información } \\
\text { de Akaike }\end{array}$ & $-4,712324$ & & $-5,086709$ & \\
\hline Criterio de Schwarz & $-3,047048$ & & $-3,421434$ & \\
\hline
\end{tabular}

Nota: EE, errores estándar en paréntesis, (); y T es el estadístico $t$ en corchetes, [].

Fuente: estimaciones de los autores a partir de los datos de Banco Mundial (2016) y GFDD Banco Mundial (2017). 


\section{Análisis de funciones de impulso-respuesta}

En este apartado se presenta el análisis dinámico de corto plazo de las funciones impulso-respuesta estimadas en un horizonte de 10 años. Estas funciones muestran la respuesta dinámica del PIB per cápita ante innovaciones - o perturbaciones - en las variables de estructura y desarrollo financiero. De acuerdo con Cuevas (2010), cada innovación debe entenderse como un aumento de una desviación estándar en la variable en cuestión, lo cual es inesperado y transitorio, pues se mantiene durante un solo periodo. Además, las respuestas son generalizadas, dado que no dependen de la ordenación de las variables del modelo.

Como ya se ha explicado, las variables de liquidez de la estructura financiera $(S A)$ y tamaño de la estructura financiera $(S S)$ son razones que evalúan la actividad bursátil con respecto a la actividad bancaria. Por lo tanto, perturbaciones positivas de ambos indicadores indicarían que la estructura financiera es cada vez más bursátil. En la Figura 3, se puede observar que la respuesta del PIB per cápita ante perturbaciones en la actividad de la estructura financiera $(S A)$ sería nula (panel a); mientras que la respuesta del PIB per cápita ante innovaciones en el tamaño de la estructura financiera $(S S)$ sería positiva hasta el tercer periodo, se diluye en el cuarto periodo y de nuevo positivo para el resto del tiempo (Figura 3, panel b). Lo anterior implicaría que si el tamaño de la estructura financiera se hiciera más bursátil tendría un efecto positivo en el crecimiento económico.

La interpretación las funciones impulso respuesta de las variables FA y FS es diferente, pues no son razones. Ahora evaluamos cómo respondería el PIB per cápita ante una perturbación positiva en la liquidez de la actividad financiera $(F A)$ o en el tamaño de la actividad financiera (FS). La Figura 3 (panel c) indica que ante un incremento inesperado de corto plazo en la liquidez del sistema financiero $(F A)$ la respuesta del PIB sería ligeramente negativa en el segundo y tercer periodo, pero ese efecto se diluirá a partir del cuarto periodo (panel c). Y, finalmente, la respuesta del PIB per cápita ante aumentos inesperados en el tamaño de la del sistema financiero (FS) sería positiva desde el segundo periodo, pero se reduciría en el cuarto periodo (panel d). Es importante diferenciar los resultados de las funciones impulso 
Méndez-Heras, Venegas-Martínez y Solis-Rosales: Finanzas y crecimiento en México...

respuestas, que son las respuestas dinámicas de corto plazo del crecimiento económico ante posibles perturbaciones en las variables financieras; de las estimaciones en los vectores de cointegración del Cuadro 7, los cuales evalúan la relación de largo plazo tomando en cuenta los valores pasados de las variables.

Figura 3. Funciones generalizadas de impulso respuesta

a. Respuesta de PIBpc ante $S A$

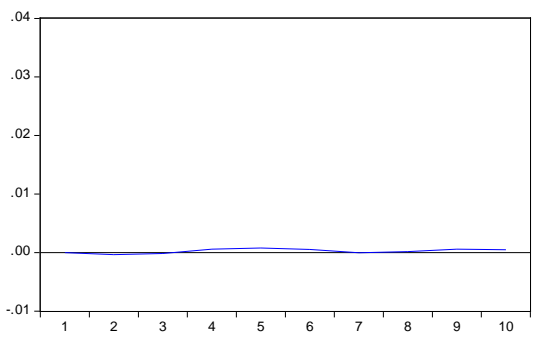

c. Respuesta de PIBpc ante FA

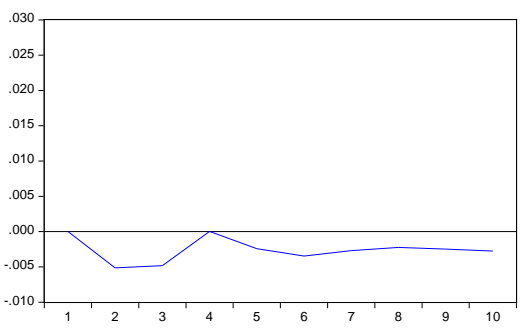

b. Respuesta de PIBpc ante $S S$

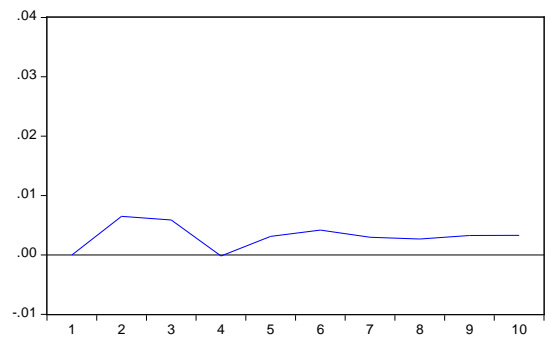

d. Respuesta de PIBpc ante FS

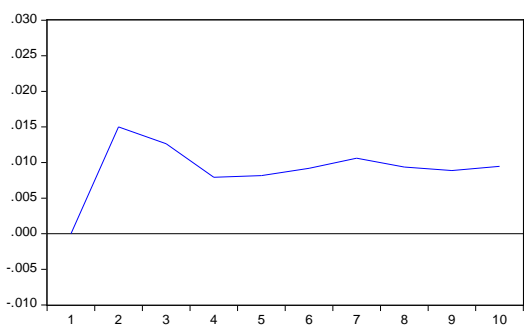

Fuente: elaboración propia con datos de Banco Mundial (2016) y GFDD Banco Mundial (2017).

\section{E. Descomposición de la varianza}

A continuación, se presenta la descomposición de los errores finales de predicción, la cual permite estudiar el comportamiento de las variables cuando se someten a perturbaciones (choques), y muestra el peso que tienen los residuos de cada variable sobre el error final de predicción del modelo. Al igual 
que con las funciones impulso-respuesta se analizan 10 años y los resultados de las variables financieras se presentan en la Tabla 8.

Tabla 8. Descomposición de la varianza

\begin{tabular}{cccc|ccc}
\hline & \multicolumn{3}{c|}{ Modelo 1 } & \multicolumn{3}{c}{ Modelo 2 } \\
\hline Periodo & PIBpc & SA & SS & PIBpc & FA & FS \\
\hline 1 & 100,00 & 0,00 & 0,00 & 100,00 & 0,00 & 0,00 \\
2 & 96,57 & 0,01 & 3,42 & 74,05 & 2,72 & 23,22 \\
3 & 95,26 & 0,01 & 4,73 & 68,42 & 3,62 & 27,96 \\
4 & 95,98 & 0,03 & 3,99 & 68,93 & 3,11 & 27,96 \\
5 & 96,13 & 0,05 & 3,82 & 70,03 & 2,92 & 27,05 \\
6 & 96,97 & 0,05 & 3,98 & 69,34 & 3,11 & 27,54 \\
7 & 96,10 & 0,05 & 3,85 & 68,21 & 3,03 & 28,76 \\
8 & 96,28 & 0,04 & 3,68 & 68,24 & 2,89 & 28,87 \\
9 & 96,32 & 0,05 & 3,63 & 68,30 & 2,83 & 28,87 \\
10 & 68,35 & 0,05 & 3,60 & 68,10 & 2,82 & 29,08 \\
\hline
\end{tabular}

Fuente: elaboración propia con datos de Banco Mundial (2016) y GFDD Banco Mundial (2017).

La descomposición de la varianza para M1 indica que los errores finales de predicción del PIB per cápita se explican en $68 \%$ por sus propias innovaciones en el periodo 10. La variable de estructura financiera $(S A)$ solo explica el $0,05 \%$ de las innovaciones del PIB per cápita en el décimo periodo, mientras que el tamaño de la estructura financiera $(S S)$ explica 3,6\% por sus propias innovaciones en el periodo 10. En cuanto a la descomposición de la varianza de los indicadores de desarrollo financiero (M2), se observa que los errores finales de predicción del PIB per cápita pasaron de $100 \%$ en el periodo uno a $68 \%$ en el periodo diez, mientras que la explicación del tamaño del sistema financiero $(F S)$ pasó de $0 \%$ a 2,82\% en el periodo diez, y la actividad financiera (FA) explicó en $29 \%$ los choques aleatorios del PIB per cápita en el último periodo. 
Méndez-Heras, Venegas-Martínez y Solis-Rosales: Finanzas y crecimiento en México...

\section{F. Prueba de cambio estructural}

Durante el periodo de estudio (1980-2014) se tiene registro de tres crisis importantes: en los años de 1980, a mediados de los años 1990 y —la más reciente- la crisis financiera internacional que tuvo efecto a partir del año 2009 en México. La prueba de cambio estructural consiste en estimar dos modelos VEC: el original, sin considerar el efecto de las crisis (1); y un segundo modelo donde se agregará una variable dummy para calcular el efecto de las crisis (2). La variable dummy tomará valores de uno en los años con crisis, y cero en el resto de los años.

La prueba de cambio estructural con la razón de verosimilitud (likelihood ratio) tiene como hipótesis el no efecto de la variable dummy y se obtiene de la siguiente forma:

$$
L R=(T-m)\left(\ln \left|\Sigma_{1}\right|-\ln \left|\Sigma_{2}\right|\right) \sim X^{2}(q),
$$

donde $T$ es el número de observaciones, $m$ es el número de parámetros (considerando los rezagos, el intercepto y la variable dummy), $\Sigma_{1}$ y $\Sigma_{2}$ son los determinantes de la matriz de covarianza residual de la ecuación original (1) y de la ecuación con el efecto de la crisis (2), respectivamente, y $q$ es el grado de libertad de la distribución asintótica chi-cuadrada (variable dummy por número de ecuaciones, que en este caso son tres). Esta prueba se realiza en el programa Eviews 9.0 (HIS Global Inc., 2015).

En esta investigación se utilizaron dos modelos, uno para medir el efecto de todo el desarrollo financiero en su conjunto y otro para ver el impacto de la estructura financiera. También se realizan dos pruebas $L R$ : en la primera, se obtuvo un valor $L R$ de 2,76 y para la segunda prueba se obtuvo un $L R$ de 1,55. Para tres grados de libertad, el valor crítico de la distribución chicuadrada, con una probabilidad de $5 \%$, es de 7,815 . Dado que los valores $L R$ son menores al valor crítico, no se puede rechazar la hipótesis nula de que las crisis no tuvieron un efecto significativo en nuestros coeficientes estimados. 


\section{Conclusiones}

En este artículo se calcularon indicadores de estructura y desarrollo financiero, y con ellos se estimaron dos modelos VEC para analizar el efecto de la estructura y la actividad financiera en el crecimiento económico de 1980 a 2014. Al considerar la liquidez del sistema financiero $(S A)$ predominó la estructura financiera bursátil, lo que coincide con Copelman (2001), quien señala que la estructura financiera de México se está haciendo más bursátil; mientras que al tomar en cuenta el tamaño de la estructura financiera (SS) predominó la estructura bancaria, lo cual coincide con los hallazgos de Beck et al. (2009) quien mostró que al utilizar indicadores de tamaño del sistema financiero predomina la actividad bancaria.

Además, se encontró que el incremento de la liquidez del sistema financiero $(F A)$ tiene un efecto positivo en el crecimiento, lo cual coincide con diversas investigaciones que encuentran una relación positiva entre las finanzas y el crecimiento económico (Beck et al., 2001; Goldsmith, 1969). Sin embargo, los resultados sugieren que el tamaño de la actividad financiera (FS) tiene un efecto negativo en el crecimiento. Este último resultado coincide con lo planteado por Beck et al. (2014), quienes advierten sobre la relación entre un sector financiero grande, que no depende de la actividad tradicional de intermediación y asociado a mayores niveles de volatilidad en el crecimiento económico.

Dentro de los resultados de este artículo, también se tiene una relación de equilibrio de largo plazo entre el PIB per cápita y los indicadores de estructura y actividad financiera $(S A, S S, F A$ y $F S)$, ya que las ecuaciones de cointegración fueron estadísticamente significativas. Este resultado va acorde con los últimos estudios que confirman una relación significativa entre la estructura financiera y el crecimiento económico (Chu, 2020; Demir \& Hall, 2017; Liu \& Zhang, 2020). Otro resultado relevante corresponde a la relación de causalidad de Granger unidireccional del PIB per cápita hacia ambos indicadores de estructura financiera, por lo que los valores pasados de la tasa de crecimiento económico explican —están correlacionados con- los valores actuales de la actividad de la estructura financiera $(S A)$ y el tamaño de la estructura financiera $(S S)$. Y —el análisis dinámico de corto plazo- 
las funciones impulso respuesta muestran que si el tamaño de la estructura financiera se hace más bursátil el efecto en el crecimiento económico será positivo.

Los resultados anteriores confirman que, durante el periodo de estudio, predominó la estructura financiera del mercado bursátil (stock-market-based) y la teoría permite hacer varias recomendaciones en materia de política regulatoria para prevenir los efectos nulos o adversos de la actividad bancaria hacia el crecimiento económico de México. En primer lugar, regular a los bancos más grandes, de manera diferente a los pequeños, con el objetivo de reducir los efectos de la alta concentración de la industria bancaria, la cual tiende a reducir los beneficios esperados en las empresas y los incentivos para innovar, acotando el efecto positivo sobre el crecimiento. En segundo lugar, crear instrumentos financieros destinados a las actividades de innovación y a la creación de empresas, ya que los bancos comerciales tienen mayor propensión a financiar empresas maduras y menos riesgosas, pero ello limita la innovación tecnológica y el aumento de la actividad económica. Y, por último, crear mecanismos para mejorar el control corporativo de las empresas sobre los bancos; ya que los bancos más grandes pueden coludirse con las empresas, haciéndolas más ineficientes, lo cual no necesariamente es lo mejor para todos los acreedores en general. 


\section{Anexo}

Tabla A1. Variables, definiciones y fuentes

\begin{tabular}{|c|c|c|}
\hline Nombre & Definición & Fuente \\
\hline PIB per cápita (PIBpc) & Logaritmo del PIB per cápita real. & Banco Mundial \\
\hline Financial-activity (FA) & $\begin{array}{l}\text { Logaritmo [Valor total de las acciones nego- } \\
\text { ciadas en el mercado bursátil como porcentaje } \\
\text { del PIB x Crédito privado otorgado por todas } \\
\text { las instituciones financieras (considera bancos y } \\
\text { otras instituciones no bancarias) como porcen- } \\
\text { taje del PIB] }\end{array}$ & GFDD Banco Mundial \\
\hline Financial-size(FS) & $\begin{array}{l}\text { Logaritmo [Capitalización de mercado como } \\
\text { porcentaje del PIB + Crédito privado otorgado } \\
\text { por todas las instituciones financieras (considera } \\
\text { bancos y otras instituciones no bancarias) como } \\
\text { porcentaje del PIB] }\end{array}$ & GFDD Banco Mundial \\
\hline Structure-activity(SA) & $\begin{array}{l}\text { Logaritmo [Valor total de las acciones negocia- } \\
\text { das en el mercado accionario como porcentaje } \\
\text { del PIB / Créditos otorgado por los bancos co- } \\
\text { merciales como porcentaje del PIB] }\end{array}$ & GFDD Banco Mundial \\
\hline Structure-size(SS) & $\begin{array}{l}\text { Logaritmo [Capitalización de mercado como } \\
\text { porcentaje del PIB/Créditos otorgado por los } \\
\text { bancos comerciales como porcentaje del PIB] }\end{array}$ & GFDD Banco Mundial \\
\hline Inflación (Inf) & $\begin{array}{l}\text { Logaritmo de } 1 \text { más la tasa porcentual de } \\
\text { inflación de la economía. }\end{array}$ & Banco Mundial \\
\hline Gobierno (Gov) & $\begin{array}{l}\text { Logaritmo del gasto de gobierno como porcen- } \\
\text { taje del PIB. }\end{array}$ & Banco Mundial \\
\hline Comercio (Com) & $\begin{array}{l}\text { Logaritmo del comercio (exportaciones más las } \\
\text { importaciones) como porcentaje del PIB. }\end{array}$ & Banco Mundial \\
\hline
\end{tabular}

Nota: se tomaron en cuenta dólares constantes de 2010. Los datos del Banco Mundial y de la OCDE corresponden a los datos de Contabilidad Nacional. Global Financial Development Database (GFDD). La construcción de los indicadores financieros basa en la metodología propuesta por Beck et al. (2001). Fuente: elaboración propia con datos de Banco Mundial (2016) y GFDD Banco Mundial (2017). 
Méndez-Heras, Venegas-Martínez y Solis-Rosales: Finanzas y crecimiento en México...

Tabla A2. Prueba de Estacionariedad conjunta. Modelo 1

Hipótesis nula: Raíz unitaria (proceso de raíz unitaria conjunta para el proceso)

\begin{tabular}{|c|c|c|}
\hline Muestra: 1980-2014 & \multicolumn{2}{|c|}{ Variables exógenas: interceptos individuales } \\
\hline \multicolumn{3}{|c|}{ Selección de rezagos basada en el criterio de Información de Schwarz (0 a 5) } \\
\hline \multicolumn{3}{|c|}{ Selección de ancho de banda con el Kernel de Newey-West } \\
\hline Observaciones totales: 191 & \multicolumn{2}{|c|}{ Sección cruzada: 6} \\
\hline Método & Estadística de prueba & Probabilidad \\
\hline Levin Lin \& Chun (probabilidades asintóticas normales) & -9.86645 & 0,000000 \\
\hline
\end{tabular}

Fuente: elaboración propia con datos de Banco Mundial (2016) y GFDD Banco Mundial (2017).

Tabla A3. Prueba de Estacionariedad conjunta. Modelo 2

Hipótesis nula: Raíz unitaria (proceso de raíz unitaria conjunta para el proceso)

\begin{tabular}{l|lc}
\hline Muestra: 1980-2014 & Variables exógenas: interceptos individuales \\
\hline \multicolumn{2}{c}{ Selección de rezagos automática basada en el criterio de Información de Schwarz (0 a 5) } \\
\hline \multicolumn{2}{c}{ Selección de ancho de banda con el Kernel de Newey-West } \\
\hline Observaciones totales: 193 & \multicolumn{2}{c}{ Sección cruzada: 6} \\
\hline Método & Estadística de prueba & Probabilidad \\
\hline Levin Lin \& Chun (probabilidades asintóticas normales) & $-7,05392$ & 0,000000 \\
\hline
\end{tabular}

Fuente: elaboración propia con datos de Banco Mundial (2016) y GFDD Banco Mundial (2017). 
Tabla A4. Prueba de normalidad de Jarque-Bera para los residuos del VEC (M1)

\begin{tabular}{|c|c|c|c|}
\hline \multicolumn{4}{|c|}{ (Hipótesis nula: los residuos tienen una distribución normal) } \\
\hline \multicolumn{4}{|c|}{ Prueba conjunta } \\
\hline & Estadístico JB & Grados de libertad & Valor de probabilidad \\
\hline & 0.945091 & 6 & 0,9876 \\
\hline \multicolumn{4}{|c|}{ Componentes individuales } \\
\hline Componente & Estadístico JB & Grados de libertad & Valor de la probabilidad \\
\hline 1 & 0,617589 & 2 & 0,7343 \\
\hline 2 & 0,213292 & 2 & 0,8988 \\
\hline 3 & 0,114210 & 2 & 0,9445 \\
\hline
\end{tabular}

Nota: se emplea el método de ortogonalización de Cholesky.

Fuente: elaboración propia con datos de Banco Mundial (2016) y GFDD Banco Mundial (2017).

Tabla A5. Prueba de normalidad de Jarque-Bera para los residuos del VEC (M2)

\begin{tabular}{|c|c|c|c|}
\hline \multicolumn{4}{|c|}{ (Hipótesis nula: los residuos tienen una distribución normal) } \\
\hline \multicolumn{4}{|c|}{ Prueba conjunta } \\
\hline & Estadístico JB & Grados de libertad & Valor de probabilidad \\
\hline & 1,886927 & 6 & 0,9298 \\
\hline \multicolumn{4}{|c|}{ Componentes individuales } \\
\hline Componente & Estadístico JB & Grados de libertad & Valor de la probabilidad \\
\hline 1 & 0,266075 & 2 & 0,8754 \\
\hline 2 & 0,869982 & 2 & 0,6473 \\
\hline 3 & 0,750870 & 2 & 0,6870 \\
\hline
\end{tabular}

Fuente: elaboración propia con datos de Banco Mundial (2016) y GFDD Banco Mundial (2017). 
Méndez-Heras, Venegas-Martínez y Solis-Rosales: Finanzas y crecimiento en México...

Tabla A6. Pruebas de LM de correlación serial multivariada

\begin{tabular}{lcc}
\hline \multicolumn{3}{c}{ (Hipótesis nula: no hay correlación serial en el orden de rezago p) } \\
\hline \multicolumn{3}{c}{ Modelo 1 (M1) } \\
\hline 1 & Estadístico LM & Valor de la probabilidad \\
2 & 15,84310 & 0,0702 \\
3 & 8,749972 & 0,4607 \\
\hline & 5,842655 & 0,7556 \\
\hline Orden de rezago $(p)$ & Estadístico LM & Valor de la probabilidad \\
\hline 1 & 10,36087 & 0,3221 \\
2 & 17,30778 & 0,0441 \\
3 & 8,924888 & 0,4442 \\
\hline
\end{tabular}

Nota: estimaciones de los autores con datos del Banco Mundial y la OCDE, LM = Multiplicador de Lagrange.

Tabla A7. Prueba de heteroscedasticidad de White para los residuos M1

\begin{tabular}{|c|c|c|c|c|c|}
\hline \multicolumn{6}{|c|}{ (Hipótesis nula de homoscedasticidad) } \\
\hline \multicolumn{2}{|c|}{ Estadístico de Chi-cuadrado (i) } & \multicolumn{2}{|c|}{ Grados de libertad } & \multicolumn{2}{|c|}{ Valor de probabilidad } \\
\hline \multicolumn{2}{|c|}{111,0396} & \multicolumn{2}{|c|}{120} & \multicolumn{2}{|c|}{0,7089} \\
\hline \multicolumn{6}{|c|}{ Componentes individuales } \\
\hline Dependiente & $R^{2}$ & $\mathrm{~F}(20,10)$ & $\begin{array}{c}\text { Valor de la } \\
\text { probabilidad }\end{array}$ & Chi-cuadrado (26) & $\begin{array}{c}\text { Valor de la } \\
\text { probabilidad }\end{array}$ \\
\hline res $1 *$ res 1 & 0,577406 & 0,683167 & 0,7757 & 17,89957 & 0,5940 \\
\hline res $2^{*}$ res 2 & 0,536407 & 0,578532 & 0,8572 & 16,62862 & 0,6769 \\
\hline res3* res3 & 0,595100 & 0,734874 & 0,7332 & 18,44811 & 0,5579 \\
\hline res $2 *$ res 1 & 0,575996 & 0,679234 & 0,7789 & 17,85588 & 0,5969 \\
\hline res $3^{*}$ res 1 & 0,601780 & 0,755587 & 0,7161 & 18,65518 & 0,5443 \\
\hline res $3 *$ res 2 & 0,463041 & 0,431170 & 0,9476 & 14,35427 & 0,8121 \\
\hline
\end{tabular}

Fuente: elaboración propia con datos de Banco Mundial (2016) y GFDD Banco Mundial (2017). 
Tabla A8. Prueba de heteroscedasticidad de White para los residuos M2

\begin{tabular}{|c|c|c|c|c|c|}
\hline \multicolumn{6}{|c|}{ (Hipótesis nula de homoscedasticidad) } \\
\hline \multicolumn{2}{|c|}{ Estadístico de Chi-cuadrado (i) } & \multicolumn{2}{|c|}{ Grados de libertad } & \multicolumn{2}{|c|}{ Valor de probabilidad } \\
\hline \multicolumn{2}{|c|}{141,2107} & \multicolumn{2}{|c|}{120} & \multicolumn{2}{|c|}{0,0904} \\
\hline \multicolumn{6}{|c|}{ Componentes individuales } \\
\hline Dependiente & R cuadrado & $\mathrm{F}(26,4)$ & $\begin{array}{c}\text { Valor de la } \\
\text { probabilidad }\end{array}$ & Chi-cuadrado (26) & $\begin{array}{c}\text { Valor de la } \\
\text { probabilidad }\end{array}$ \\
\hline res $1{ }^{*}$ res 1 & 0,806604 & 2,085375 & 0,1161 & 25,00474 & 0,2012 \\
\hline res $2 *$ res 2 & 0,735918 & 1,393351 & 0,3009 & 22,81346 & 0,2980 \\
\hline res3* res3 & 0,792592 & 1,910706 & 0,1463 & 24,57035 & 0,2184 \\
\hline $\operatorname{res} 2 *$ res 1 & 0,908048 & 4,937591 & 0,0065 & 28,14947 & 0,1059 \\
\hline res $3^{*}$ res 1 & 0,814268 & 2,192057 & 0,1011 & 25,24232 & 0,1923 \\
\hline res $3{ }^{*}$ res 2 & 0,639548 & 0,887149 & 0,6094 & 19,82600 & 0,4689 \\
\hline
\end{tabular}

Fuente: elaboración propia con datos de Banco Mundial (2016) y GFDD Banco Mundial (2017).

Tabla A9. Raices inversas del polinomio auto regresivo característico
A. Modelo (M1)
B. Modelo 2 (M2)
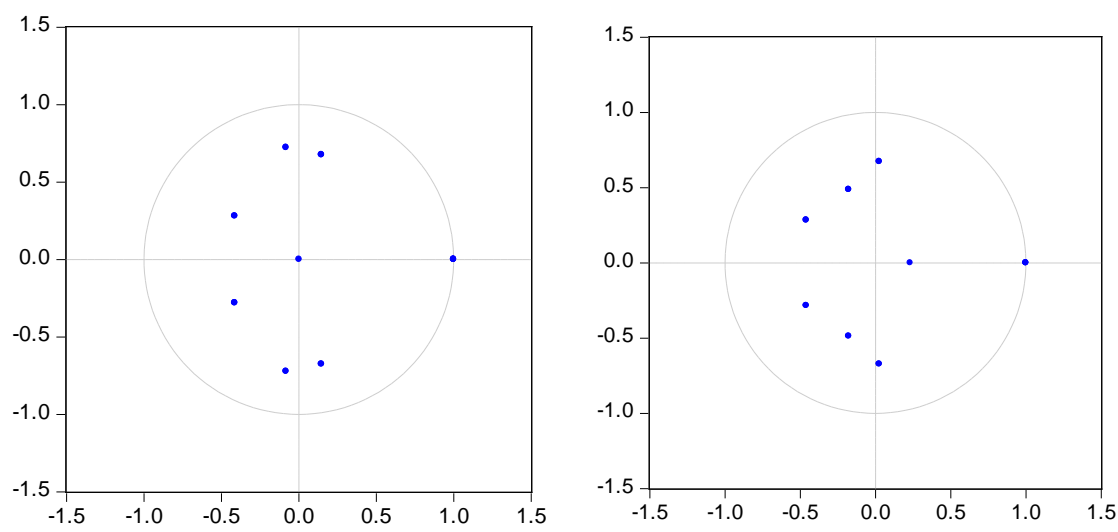

Fuente: elaboración propia con datos de Banco Mundial (2016) y GFDD Banco Mundial (2017). 
Méndez-Heras, Venegas-Martínez y Solis-Rosales: Finanzas y crecimiento en México...

\section{Referencias}

Acemoglu, D. \& Robinson, J. A. (2013). Why Nations Fail, The Origins of Power, Prosperity and Poverty. Penguin Random House.

Aiyar, S., \& Ebeke, C. (2020). Inequality of Opportunity, Inequality of Income and Economic Growth. World Development, 136, 105115. ht tps://doi.org/10.1016/j.worlddev.2020.105115

Allen, F., \& Carletti, E. (2008). The Role of Liquidity in Financial Crises [working paper]. http://dx.doi.org/10.2139/ssrn.1268367

Arestis, P., Demetriades, P., \& Luintel, K. B. (2001). Financial Development and Economic Growth: The Role of Stock Markets. Journal of Money, Credit and Banking, 33(1), 16-41. https://doi.org/10.2307/2673870

Bagehot, W. (1873). Lombard Street: A Description of the Money Market. Henry S. King.

Banco Mundial (2017). Global Financial Development Database. https: //www.worldbank.org/en/publication/gfdr/data/global-financialdevelopment-database

Banco Mundial (2016). World Development Indicators. http://data.worldba nk.org/data-catalog/world-development-indicators

Barro R. (1991). Economic growth in a cross section of countries. Quarterly Journal of Economics, 106 (2), 407-443. https://doi.org/10.2307/2937 943

Beck, T,. Demirgüç-Kunt, A., Levine, R., \& Maksimovic, V. (2001). Financial Structure and Economic Development: Firm, Industry, and Country Evidence. En A. Demirgüç-Kunt, \& R. Levine (eds), Financial Structure and Economic Growth (pp. 189-241), The MIT Press.

Beck, T., Demirgüç-Kunt, A., \& Levine, R. (2009). Financial Institutions and Markets across Countries and over Time, Data and Analysis [Policy Research Working Paper, No. 4943]. Development Research Group, Finance and Private Sector Team. The World Bank. https://openkn owledge.worldbank.org/bitstream/handle/10986/4154/WPS4943.pdf 
Beck, T., Degryse, H., \& Kneer, C. (2014). Is More Finance Better? Disentangling Intermediation and Size Effects of Financial Systems. Journal of Financial Stability, 10, 50-64. https://doi.org/10.1016/j. jfs.2013.03.005

Cárdenas, E. (2015). El largo curso de la economía mexicana: de 1780 a nuestros días. Fondo de Cultura Económica.

Chu, L. K. (2020). Financial Structure and Economic Growth Nexus Revisited. Borsa Istanbul Review, 20(1), 24-36. https://doi.org/10.1 016/j.bir.2019.08.003

Clavellina Miller, J. L. (2013). Crédito bancario y crecimiento económico en México. Economía Informa, 378, 14-36. https://doi.org/10.1016/S018 5-0849(13)71306-9

Colpeman, M. (2000). Financial Structure and Economic Activity in Mexico. Center of Analysis and Economic Research [document de trabajo, Conferencia sobre estructura financiera y desarrollo económico, Washington, DC, Banco Mundial (Vol. 10]. http://citeseerx.ist.psu.edu/viewdoc/do wnload?doi=10.1.1.19.1052\&rep=rep $1 \&$ type $=$ pdf

Cuevas, V. (2010). México: dinámica de las exportaciones manufactureras. Revista Cepal, (102), 153-174. https://repositorio.cepal.org/bitstrea $\mathrm{m} / \mathrm{handle} / 11362 / 37386 / 1 / \mathrm{RVE} 102$ _es.pdf

Cuevas, V., \& Calderón, C. (2019). Crecimiento industrial y aumento de los precios de los bienes de consumo en México: un análisis econométrico. Revista de la CEPAL, (129), 191-215. https://www.cepal.org/es/publi caciones/45012-crecimiento-industrial-aumento-precios-bienes-con sumo-mexico-un-analisis

Cuevas, V., \& Calderón, C. (2020). Human Capital Formation and Economic Growth Across the World: A Panel Data Econometric Approach. Economía, Sociedad y Territorio, 20(62), 25-54. https://do i.org/10.22136/est20201466 
Méndez-Heras, Venegas-Martínez y Solis-Rosales: Finanzas y crecimiento en México...

De Gregorio, J., \& Guidotti, P. E. (1995). Financial Development and Economic Growth. World Development, 23(3), 433-448. https://doi. org/10.1016/0305-750X(94)00132-I

Demetriades, P. O., \& Rewilak, J. M. (2020). Recovering the finance-growth nexus. Economics Letters, 196, 109563. https://doi.org/10.1016/j.econ let.2020.109563

Demirgüç-Kunt A., \& Levine, R. (1996). Stock Markets, Corporate Finance and Economic Growth: An Overview. The World Bank Economic Review, 10(2), 223-239. https://doi.org/10.1093/wber/10.2.223

Demirgüç-Kunt A., \& Levine, R. (2001). Financial Structure and Economic Growth: Perspective and Lessons. En A. Demirgüç-Kunt, \& R. Levine (eds), Financial Structure and Economic Growth (pp. 2-14), The MIT Press.

Demir, A. U., \& Hall, S. G. (2017). Financial Structure and Economic Development: Evidence on the View of 'New Structuralism'. International Review of Financial Analysis, 52(C), 252-259. https://doi.org/10.1016/ j.irfa.2017.07.003

Ejea, G., Garrido, C., Leriche, C., \& Quintana, E. (1991). Mercado de Valores, Crisis y Nuevos Circuitos Financieros en México: 1970-1990. Universidad Autónoma Metropolitna-Azcapotzalco.

Garrido, C., \& Martínez Pérez, J. F. (2004). El sistema financiero mexicano, Evolución reciente y perspectivas. El Cotidiano, 19(123),19-29. https: //www.redalyc.org/pdf/325/32512303.pdf

Goldsmith, R. (1969). Financial Structure and Development. Yale University Press.

Gerschenkron, A. (1962). Economic Backwardness in Historical Perspective: A Book of Essays. Harvard University Press.

Hamilton, J. (1994). Time Series Analysis, Princeton, Princeton University Press. 
Hernández, F., \& Villagómez, A. (2013). El enigmático sistema bancario mexicano contemporáneo. Centro de Estudios Espinosa Yglesias.

Hicks, J. R. (1969). A Theory of Economic History. Clarendon Press.

HIS Global Inc. (2015). EVIEWS 9 User's Guide II. HIS Global Inc.

Johansen, S. (1995). Likelihood-based Inference in Cointegrated Vector Autoregressive Models, Oxford University Press.

Khan, R., \& Chaudhry, I. S. (2019). Impact of Human Capital on Employment and Economic Growth in Developing Countries. Review of Economics and Development Studies, 5(3), 487-496. https://doi.org/ 10.26710/reads.v5i3.701

Kim, D. H., Lin, S. C., \& Chen, T. C. (2016). Financial Structure, Firm Size and Industry Growth, International Review of Economics and Finance, 41, 23-39. https://doi.org/10.1016/j.iref.2015.10.002

Levine, R. (1997). Financial Development and Economic Growth: Views and Agenda. Journal of Economic Literature, 35 (2), 688-726. https: //www.jstor.org/stable/2729790

Levine, R., (2002). Bank-Based or Market-Based Financial Systems: Which is better? Journal of Financial Intermediation, 11(4), 398-428. https: //doi.org/10.1006/jfin.2002.0341

Levine, R., (2005). Finance and Growth: Theory and Evidence. En Aghion, A. \& S. N. Durlauf (eds), Handbook of Economic Growth, 1, Part A. https://doi.org/10.1016/S1574-0684(05)01012-9

Liu, G., \& Zhang, C. (2020). Does Financial Structure Matter for Economic Growth and Income Inequality in China. China Economic Review, 61, 101194. https://doi.org/10.1016/j.chieco.2018.06.006

Marichal, C. (2010). Nueva historia de las grandes crisis financieras: una perspectiva global 1873-2008. Sudamericana.

Méndez-Heras, L., \& Ongena, S. (2020). "Finance and Growth" Re-Visited. Journal of Financial Management, Markets and Institutions, 8(01), 1-28. https://doi.org/10.1142/S2282717X20500012 
Méndez-Heras, Venegas-Martínez y Solis-Rosales: Finanzas y crecimiento en México...

Negrín, J.L., Ocampo, D., \& Struck, P. (2010). Competencia en el mercado de crédito bancario mexicano. En A. Castañeda (coord.). Los grandes problemas de México: microeconomía (37-75). El Colegio de México.

Ortiz, C. H., Jiménez D. M., \& Cruz, G. (2019). El impacto de la infraestructura en el crecimiento económico colombiano. Lecturas de Economía, (90), 97-126. https://doi.org/10.17533/udea.le.n90a04

Ortiz, E., Cabello, A., \& López Herrera, F. (2007), Mercados de capital emergentes y desarrollo e integración. En A. Giron. \& E. Correa (eds). Del Sur hacia el Norte: Economía politica del orden económico internacional emergente (pp. 128-139). Consejo Latinoamericano de Ciencias (CLACSO). http://biblioteca.clacso.edu.ar/clacso/sur-su r/20100705070121/13Ortiz-Cabello-LopezH.pdf

Rajan, R., \& Zingales L. (1998). Financial Dependence and Growth. American Economic Review, 88(3), 559-586. https://www.jstor.org/ stable/116849

Sánchez, A. (2006). Crecimiento económico, desigualdad y pobreza: una reflexión a partir de Kuznets. Problemas del desarrollo, 37(145), 11-30. https://www.redalyc.org/pdf/118/11820086002.pdf

Salazar-Nuñez, H., \& Venegas-Martínez, F. (2018). Impacto del uso de energía y formación bruta de capital en el crecimiento económico. Un análisis de datos de panel en 73 países agrupados por nivel de ingreso y producción de petróleo, El Trimestre Económico, LXXXV (2), 341-364. https://doi.org/10.20430/ete.v85i338.342

Shumpeter, J. A. (1911). Theorie der wirtschaftlichen Entwicklung. Kyklos.

Silva, T. C., Tabak, B. M., \& Laiz, M. T. (2021). The Finance-growth Nexus: The Role of Banks. Economic Systems, 45(1), 100762. https://doi.org/ 10.1016/j.ecosys.2020.100762

Solow, R. (1956). A Contribution to the Theory of Economic Growth. The Quarterly Journal of Economics, 70(1). 65-94. https://doi.org/10.2307/ 1884513 
Stiglitz, J. (1985). Credit Markets and the Control of Capital. Journal of Money, Credit and Banking, 17(2), 133-152. https://doi.org/10.230 7/1992329

Tadesse, S. (2002). Financial Architecture and Economic Performance: International Evidence. Journal of Financial Intermediation, 11, 429454. https://doi.org/10.1006/jfin.2002.0352

Tongurai, J., \& Vithessonthi, C. (2018). The Impact of the Banking Sector on Economic Structure and Growth. International Review of Financial Analysis, 56(C), 193-207. https://doi.org/10.1016/j.irfa.2018.01.002 